University of Nebraska - Lincoln

DigitalCommons@University of Nebraska - Lincoln

USDA Wildlife Services - Staff Publications

U.S. Department of Agriculture: Animal and Plant Health Inspection Service

9-2019

\title{
Predicting functional responses in agro-ecosystems from animal movement data to improve management of invasive pests
}

\author{
Mark Q. Wilber \\ Colorado State University \& National Wildlife Research Center, Fort Collins \\ Sarah M. Chinn \\ University of Georgia, sarahchinn@uga.edu \\ James C. Beasley \\ University of Georgia \\ Raoul K. Bourghton \\ University of Florida \\ Ryan K. Brooks \\ Follow this and additional works at: https://digitalcommons.unl.edu/icwdm_usdanwrc \\ (1) \\ Part of the Natural Resources and Conservation Commons, Natural Resources Management and \\ Policy Commons, Other Environmental Sciences Commons, Other Veterinary Medicine Commons, \\ See next page for additional authors \\ Population Biology Commons, Terrestrial and Aquatic Ecology Commons, Veterinary Infectious Diseases \\ Commons, Veterinary Microbiology and Immunobiology Commons, Veterinary Preventive Medicine, \\ Epidemiology, and Public Health Commons, and the Zoology Commons
}

Wilber, Mark Q.; Chinn, Sarah M.; Beasley, James C.; Bourghton, Raoul K.; Brooks, Ryan K.; Ditchkoff, Stephen S.; Fischer, Justin W.; Hartley, Steve B.; Holmstrom, Lindsey K.; Kilgo, John C.; Lewis, Jesse S.; Miller, Ryan S.; Snow, Nathan P.; VerCauteren, Kurt C.; Wisely, Samantha M.; Webb, Colleen T.; and Peen, Kim M., "Predicting functional responses in agro-ecosystems from animal movement data to improve management of invasive pests" (2019). USDA Wildlife Services - Staff Publications. 2321.

https://digitalcommons.unl.edu/icwdm_usdanwrc/2321

This Article is brought to you for free and open access by the U.S. Department of Agriculture: Animal and Plant Health Inspection Service at DigitalCommons@University of Nebraska - Lincoln. It has been accepted for inclusion in USDA Wildlife Services - Staff Publications by an authorized administrator of DigitalCommons@University of Nebraska - Lincoln. 


\section{Authors}

Mark Q. Wilber, Sarah M. Chinn, James C. Beasley, Raoul K. Bourghton, Ryan K. Brooks, Stephen S.

Ditchkoff, Justin W. Fischer, Steve B. Hartley, Lindsey K. Holmstrom, John C. Kilgo, Jesse S. Lewis, Ryan S. Miller, Nathan P. Snow, Kurt C. VerCauteren, Samantha M. Wisely, Colleen T. Webb, and Kim M. Peen 


\title{
Predicting functional responses in agro-ecosystems from animal movement data to improve management of invasive pests
}

\author{
Mark Q. Wilber (D),${ }^{1,2}$ Sarah M. Chinn (D), 3,12 James C. Beasley $(D),{ }^{3}$ Raoul K. Boughton, ${ }^{4}$ Ryan K. Brook, ${ }^{5}$

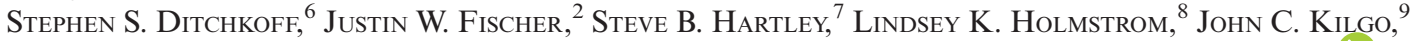 \\ Jesse S. Lewis, ${ }^{10}$ Ryan S. Miller, ${ }^{8}$ Nathan P. Snow, ${ }^{2}$ Kurt C. VerCauteren, ${ }^{2}$ Samantha M. Wisely (iD ${ }^{11}$ \\ Colleen T. Webb, ${ }^{1}$ and Kim M. PePin (iD ${ }^{2}$ \\ ${ }^{1}$ Department of Biology, Colorado State University, Fort Collins, Colorado 80523 USA \\ ${ }^{2}$ USDA, Animal and Plant Health Inspection Service, Wildife Services, National Wildlife Research Center, Fort Collins, Colorado \\ 80521-2154 USA \\ ${ }^{3}$ Savannah River Ecology Laboratory, Warnell School of Forestry and Natural Resources, University of Georgia, P.O. Drawer E, \\ Aiken, South Carolina 29081 USA \\ ${ }^{4}$ Wildlife Ecology and Conservation, Range Cattle Research and Education Center, University of Florida, Ona, Florida 33865 USA \\ ${ }^{5}$ Department of Animal \& Poultry Science and Indigenous Land Management Institute, College of Agriculture and Bioresources, \\ University of Saskatchewan, Saskatoon, Saskatchewan S7N 5 AB Canada \\ ${ }^{6}$ School of Forestry and Wildlife Sciences, Auburn University, Auburn, Alabama 36849 USA \\ ${ }^{7}$ U.S. Geological Survey, Wetland and Aquatic Research Center, 700 Cajundome Boulevarde, Lafayette, Louisiana 70506 USA \\ ${ }^{8}$ Center for Epidemiology and Animal Health, USDA Animal \& Plant Health Inspection Service, Fort Collins, Colorado 80526 USA \\ ${ }^{9}$ USDA Forest Service Southern Research Station, P.O. Box 700, New Ellenton, South Carolina 29809 USA \\ ${ }^{10}$ College of Integrative Sciences and Arts, Arizona State University, Mesa, Arizona 85212 USA \\ ${ }^{11}$ Department of Wildlife Ecology and Conservation, University of Florida, Gainesville, Florida 32611 USA
}

Citation: Wilber, M.Q, S. M. Chinn, J. C. Beasley, R. K. Boughton, R. K. Brook, S. S. Ditchkoff, J. W. Fischer, S. B. Hartley, L. K. Holmstrom, J. C. Kilgo, J. S. Lewis, R. S. Miller, N. P. Snow, K. C. VerCauteren, S. M. Wisely, C. T. Webb, and K. M. Pepin. 2020. Predicting functional responses in agro-ecosystems from animal movement data to improve management of invasive pests. Ecological Applications 30 (1):e02015. 10.1002/eap.2015

Abstract. Functional responses describe how changing resource availability affects consumer resource use, thus providing a mechanistic approach to prediction of the invasibility and potential damage of invasive alien species (IAS). However, functional responses can be context dependent, varying with resource characteristics and availability, consumer attributes, and environmental variables. Identifying context dependencies can allow invasion and damage risk to be predicted across different ecoregions. Understanding how ecological factors shape the functional response in agro-ecosystems can improve predictions of hotspots of highest impact and inform strategies to mitigate damage across locations with varying crop types and availability. We linked heterogeneous movement data across different agro-ecosystems to predict ecologically driven variability in the functional responses. We applied our approach to wild pigs (Sus scrofa), one of the most successful and detrimental IAS worldwide where agricultural resource depredation is an important driver of spread and establishment. We used continentalscale movement data within agro-ecosystems to quantify the functional response of agricultural resources relative to availability of crops and natural forage. We hypothesized that wild pigs would selectively use crops more often when natural forage resources were low. We also examined how individual attributes such as sex, crop type, and resource stimulus such as distance to crops altered the magnitude of the functional response. There was a strong agricultural functional response where crop use was an accelerating function of crop availability at low density (Type III) and was highly context dependent. As hypothesized, there was a reduced response of crop use with increasing crop availability when non-agricultural resources were more available, emphasizing that crop damage levels are likely to be highly heterogeneous depending on surrounding natural resources and temporal availability of crops. We found significant effects of crop type and sex, with males spending $20 \%$ more time and visiting crops $58 \%$ more often than females, and both sexes showing different functional responses depending on crop type. Our application demonstrates how commonly collected animal movement data can be used to understand context dependencies in resource use to improve our understanding of pest foraging behavior, with implications for prioritizing spatiotemporal hotspots of potential economic loss in agro-ecosystems.

Manuscript received 23 April 2019; revised 22 August 2019; accepted 4 September 2019. Corresponding Editor: David S. Schimel.

${ }^{12}$ Corresponding Author. E-mail: sarahchinn@uga.edu 
Key words: agro-ecosystems; continuous-time functional movement models; crop damage; functional response; habitat; invasive alien species; movement ecology; resource selection; wild pigs (Sus scrofa).

\section{INTRODUCTION}

The manner in which invasive alien species (IAS) select resources affects the extent of ecological and economic damage they cause (Vitousek et al. 1997, Pimental 2007, Shwiff et al. 2012). At a fundamental level, resources are exploited by consumers, IAS or otherwise, depending on a functional response, which describes the relationship between intake rate of a consumer and resource density or availability (functional response; Holling 1959, Murdoch and Oaten 1975). There are three general families of functional responses: a linear functional response (Type I), a hyperbolically saturating functional response (Type II), and a sigmoidally saturating functional response (Type III; Fig. 1; Holling 1959). These functional responses can reflect different underlying foraging behaviors, have different effects on predicted stability of ecological systems at equilibrium, and determine the ability of a species to invade a system (Murdoch and Oaten 1975, Van Leeuwen et al. 2007). Studies on the functional response of invasive species have led to an emerging paradigm in invasion ecology that uses functional responses to predict the impact of IAS upon invasion (Dick et al. 2014, 2017; but see Vonesh et al. 2017). However, functional responses of IASs are typically measured in the lab and with a single resource (where resource density is manipulated and resource consumption is measured, e.g., Holling 1959), which can ignore realistic context dependencies present in nature (Dick et al. 2014, Paterson et al. 2015).

In contrast, resource selection analyses often consider functional responses at the landscape level (henceforth landscape functional response), where the relationship between the proportion of available and used resource in an animal's home range is quantified (e.g., Mysterud and Ims 1998, Godvik et al. 2009). One disadvantage of landscape functional responses is they typically do not directly measure resource consumption, but rely on proxies of resource use, such as time spent in a resource (Mysterud and Ims 1998). An advantage of landscape functional responses is that covariates associated with context dependencies in resource use, such as availability of multiple resource types, can be measured in the field to highlight potential mechanisms affecting resource selection (Mysterud and Ims 1998). Landscape functional responses are well suited to examine four general factors that can affect the functional responses with respect to a focal resource: focal resource characteristics, non-focal resource availability, consumer attributes, and environmental variables (Holling 1959, Dick et al. 2014). In terms of IAS, understanding how these four factors affect landscape functional responses can improve our ability to predict their potential impact under different ecological conditions.
Landscape functional responses, and other metrics of resource use, are typically quantified using spatial animal movement data (Mysterud and Ims 1998, Manly et al. 2007). However, between-study differences in the way that movement data are collected (e.g., animal locations are obtained at different time scales) can make it difficult to quantitatively compare resource use across studies. This is particularly problematic when linking animal movement data from multiple studies to functional responses as the rate of resource use is inherently time dependent. Recent advances in continuous-time movement models (Buderman et al. 2016, Hooten and Johnson 2017) can standardize the time scale of movement trajectories across individuals, while accounting for the uncertainty in the animal's true location. Importantly, this allows one to test the drivers of landscape functional responses under the variability of ecological conditions spanned by multiple studies, providing a unique opportunity to link context dependencies in the landscape functional response to the mechanisms underlying resource use and damage.

Agro-ecosystems are ideal for examining how ecological and environmental conditions affect landscape functional responses because they comprise a complex matrix of native ecosystems and agricultural crops. Agricultural crops are a seasonally variable resource, which provide abundant calories, protein, and essential fatty acids for wildlife, making them particularly vulnerable to damage and consumption (Putman and Moore 1998, Shwiff et al. 2012). Crop availability in time and space is typically documented in great detail. For example, the National Agricultural Statistics Service (NASS; USDA 2018a) measures the yearly location of agricultural resources at a $30 \times 30 \mathrm{~m}$ scale across the continental United States. This provides an unparalleled measure of "availability" of food resources for wildlife, allowing quantification of how availability of crop resources affects use by wildlife species, i.e., the landscape functional response. For IAS, this landscape agricultural functional response can influence the invasibility of an agro-ecosystem, and the potential impact a species may have on agriculture upon invasion (Lonsdale 1999, Snow et al. 2017a). Moreover, understanding how ecological factors influence the agricultural functional response can improve predictions of hotspots of highest impact and inform strategies to mitigate crop damage across locations with varying crop types and availability.

We examined the landscape agricultural functional response (hereafter agricultural functional response) of invasive wild pigs (Sus scrofa) using movement data collected across multiple studies spanning the national level in the United States. Wild pigs are one of the most destructive large mammals globally and often have significant ecological impacts on the ecosystems they 


\section{From movement data to crop use}

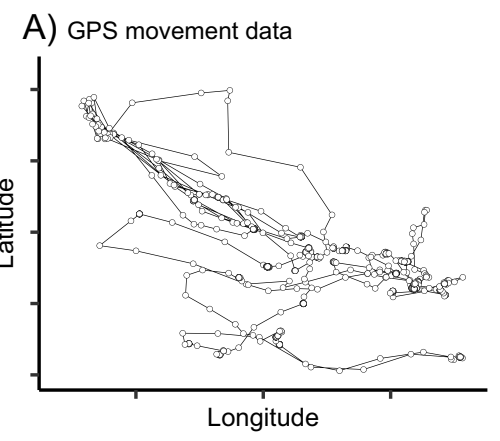

B) Fit continuous-time movement model

C) Rasterize movement trajectory on agricultural layer
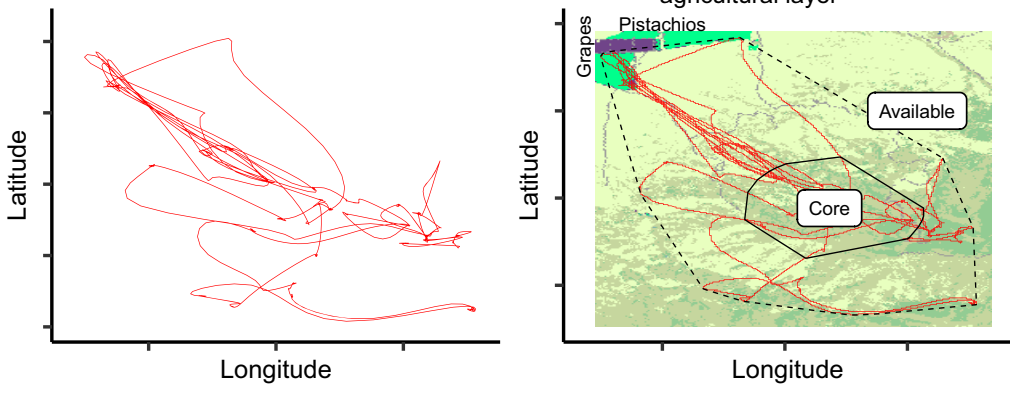

2. Extracting monthly crop use

D
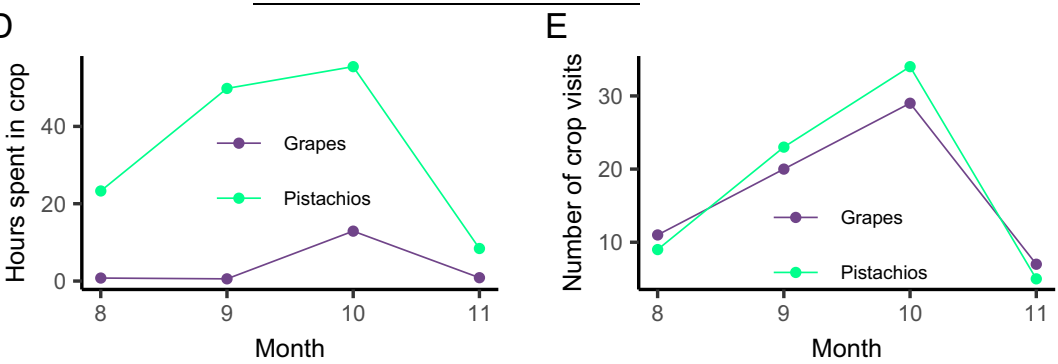

3. From crop use to the agricultural functional response

F) Monthly use defines the functional response

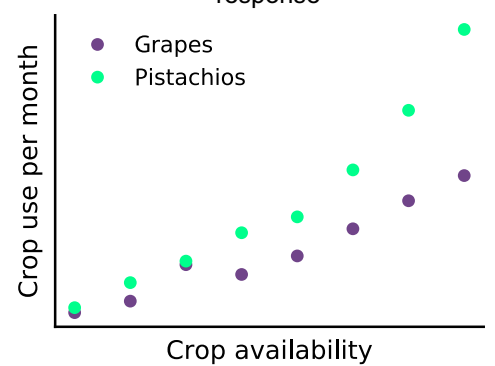

$\mathrm{G})$ Shape of the functional response

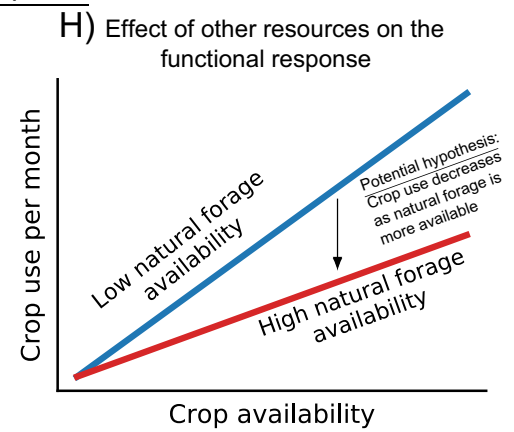

FIG. 1. To compute the agricultural functional response for wild pigs $(n=326)$, we (A) obtained GPS movement data, (B) fit a continuous-time functional movement model to the data to place all wild pig movements on the same time scale (Buderman et al. 2016), and (C) rasterized the continuous-time movement trajectory onto an agricultural data layer. Using this rasterized, continuous-time movement data, we computed (D) the amount of time a wild pig spent in particular crop types per month, (E) the number of visits to crops per month, and additional covariates in the core home range and area available to the wild pig. We used the crop use data to $(\mathrm{F})$ define the functional response to answer two questions: $(\mathrm{G})$ what is the shape of the agricultural functional response for wild pigs and $(\mathrm{H})$ what is the effect of non-agricultural forage resources on this functional response?

invade (Barrios-García and Ballari 2012, Bevins et al. 2014, Mayer and Beasley 2017). As omnivores with a generalist diet, wild pigs exploit an array of food resources, including agricultural crops in both their native and invasive ranges (Ballari and Barrios-García 2014). In Canada, wild pigs are spreading rapidly and use a wide range of agricultural crops (Brook and Van Beest 2014). Throughout North America, where wild pigs are invasive and rapidly spreading (Snow et al. 2017a) despite substantial investment on control (Pepin et al. 2019), depredation of agricultural resources may play a crucial role in their expansion and establishment (Tolleson et al. 1995, Paolini et al. 2018). However, crop selection and usage behavior of wild pigs in agro-ecosystems remain poorly understood, making it challenging to predict how crop availability might impact wild pig invasion success.

An understanding of agricultural functional responses of IASs is an important stepping-stone towards better prediction of IAS expansion in highly heterogeneous agro-ecosystems. In comparison to species distribution models that predict, for example, the probability of observing an IAS in a crop field given surrounding covariates, understanding the shape and context dependencies of the agricultural functional response in IASs directly relates to the foraging mechanisms underlying 
IAS crop use and how these mechanisms are affected by ecological and environmental factors on the landscape. Thus, species distribution models are phenomenological, while functional response models are mechanistic. In general, accurate mechanistic models will be more robust in different ecological conditions because they describe an understanding of how ecological processes interact rather than relying solely on "in-sample" patterns for prediction.

We hypothesized that wild pigs would selectively use crops more often when natural forage resources were low. We tested two predictions regarding the agricultural functional response. First, we tested whether the shape of the agricultural functional response was more consistent with a Type II or Type III functional response at low crop availability. When crops are available, a Type II response would predict that pigs immediately forage optimally, whereas a Type III response predicts that pigs would slowly increase selection as crop densities increase. In both types of functional responses, saturation occurs at high crop density, in which foraging behavior is constant despite increasing crop density. We predicted wild pigs would preferentially use nonagricultural resources until crops became nutritionally profitable to exploit despite possible risks such as predation or hunting in crop fields and would thus follow a Type III functional response. Building on this expectation, we hypothesized that the availability of non-agricultural forage resources on the landscape, such as increased primary productivity, would reduce use of agricultural resources (Fournier-Chambrillon et al. 1995, Ballari and Barrios-García 2014). We tested this second prediction by examining how availability of nonagricultural forage resources affected the functional response of wild pigs, after controlling for effects of agricultural resource attributes, pig attributes, and environmental characteristics on the functional response. We expected that increasing the availability of non-agricultural resources would reduce wild pig selection of crops, despite increased crop availability. Our application demonstrates how commonly collected animal movement data can be used to understand context dependencies in resource use to improve our understanding of pest foraging behavior and ability to predict IAS expansion.

\section{Materials And Methods}

\section{National-scale wild pig movement data}

We used global positioning system (GPS) data collected from 326 wild pigs in the United States, of which 52 were in California, 76 in Florida, 94 in Texas, 21 in Missouri, 30 in Georgia, 22 in Louisiana, and 31 in South Carolina (See Appendix S1: Section S1, Fig. S1). Data were from 24 different studies conducted between May 2005 and November 2017 for different lengths of time and purposes; thus, individuals were collared for varying amounts of time and GPS fix rates varied among studies (e.g., 95\% of fixes ranged from 15 to 60 min between each GPS location, see Appendix S1: Section S1 for details).

We standardized GPS fix rates across wild pigs and studies by fitting continuous-time, functional movement models (FMM) to observed GPS data for a given individual (Fig. 1; Buderman et al. 2016, Hooten et al. 2017). The FMM used basis functions to fit a phenomenological, continuous-time, movement model to a set of discrete GPS fixes (Fig. 1; Appendix S1: Section S2; Buderman et al. 2016), such that we could predict wild pig locations, with uncertainty, at any time between the start and end of the tracking period (Appendix S1: Section S2). By using true locations at the common fix-rate time interval across studies, in this case two hours, we did not have the ability estimate time spent in crops and the number of visits to crops because observing a GPS fix in a crop does not, on its own, provide information on the velocity of the individual. Without such velocity information, which the Buderman method provides, the ability to estimate time spent in crops or number of crop visits, and thus predict the functional response, would be severely limited. Moreover, restricting observations to a two-hour time interval would require exclusion of data for some individuals where there were more fine-scale GPS fixes.

\section{Defining use and availability of agricultural crops}

We used $30 \times 30 \mathrm{~m}$ scale agricultural raster data available from NASS across the contiguous United States, where each pixel specified the primary type of crop in that area, including land cover types when there were no crops (Appendix S1: Section S3; USDA 2018a). These agricultural raster data varied by year based on NASS surveys. We defined 10 different crop groups based on general similarities in crop type and caloric content that were used in the following analyses (Appendix S1: Table S1): cereals, fruit and nuts, sugar, oilseeds, beverages and spices, roots and tubers, vegetables and melons, legumes, tobacco, and other (consisting of only cotton in this study).

We defined crop use for a particular crop type as the total time a wild pig spent in a crop type divided by the total time for which a wild pig was collared in a given month for a given year (Fig. 1). The total time of crop use provides an overall metric of use for linking crop use to crop damage in future studies. Total time of crop use can also be decomposed into two processes that can provide additional insight into the mechanisms underlying crop use: monthly visitation rate of wild pigs to crop fields and average time spent in a crop field per visit. For example, two pigs could have the same total time of crop use, but one may have only visited a crop field once for a long period of time while another visited a crop field multiple times for shorter periods of time: distinctly different foraging behavior. Moreover, the monthly 
visitation rate of wild pigs to crop fields is closely analogous to a traditional consumer-resource functional response that describes how the rate of consumer contact and allocation of a resource varies with resource density (Murdoch and Oaten 1975). Therefore, we also analyzed monthly crop visitation rate and average time spent in a crop field per visit as metrics of crop use. We defined a crop visit as any time a pig entered a crop field within a month (Fig. 1). Visits to crop fields were considered distinct if they were separated by $\geq 30 \mathrm{~min}$ (Appendix S1: Section S4). We defined monthly visitation rate as the number of visits per month divided by the monthly time collared. In Appendix S1: Section S5, we analyzed average time spent in a crop field per visit.

We defined crop availability for a given wild pig as the total area of the crop within the area available for foraging. We defined the area available to a wild pig as the $100 \%$ minimum convex polygon (MCP) that encompassed all that individual's GPS fixes (for details see Appendix S1: Section S6; Thurfjell et al. 2009). While there are alternatives for defining available area including buffering the $100 \%$ MCP by some amount (Northrup et al. 2013), drawing a circle of available area centered at the center of an animal's observed locations with a radius equal to the farther observed location (i.e., capture radius; Bond et al. 2016), and using a utilization distribution of an animal (Bond et al. 2016), we aimed to ensure inclusion of all the potential area that was available to each individual within its home range. We chose the $100 \% \mathrm{MCP}$ as it is an intermediate estimate of available area compared to the capture radius and utilization distribution (Bond et al. 2016). We defined the area available for a particular crop type as the area of the crop available divided by the total area available.

Two other crop characteristics could also affect crop use: the distance a wild pig had to travel to crop patches and the seasonal availability of crops. We defined the distance to crops as the mean distance between the center of a wild pig's core home range ( $50 \% \mathrm{MCP})$ to the center of crop fields available (Appendix S1: Section S3). For crop seasonality, we used the most active planting and harvest date range (the period when $15-85 \%$ of the crop was planted or harvested, based on $20 \mathrm{yr}$ of historical crop progress estimates; e.g., USDA 2010) to delineate a time period over which a particular crop was available or not available for foraging (additional details in Appendix S1: Section S3).

\section{Non-agricultural covariates}

We used the Enhanced Vegetation Index (EVI) as a general metric for plant productivity and non-agricultural forage availability (Pettorelli et al. 2005, 2011). As wild pig diet consists primarily of herbaceous forage (Ballari and Barrios-García 2014), vegetation indices provide a proxy for the availability of herbaceous forage that is consistently measured across a large spatial scale. For each wild pig, we computed the mean monthly EVI in the individual's core home range, excluding any agricultural land within the core home range from the calculation. Greater values of mean monthly EVI were a proxy for greater availability of non-agricultural forage resources (Appendix S1: Section S3). We also included canopy cover near crop fields as an environmental factor that could affect crop use by providing cover for wild pigs within range of a field (Appendix S1: Section S3; Amici et al. 2012). Hard mast is also an important component of wild pig diets but is highly variable in space and time. While detailed historical masting data are collected locally at some sites, there was no large-scale, historical hard masting database across the United States. Because of these challenges, we did not explicitly include masting as an additional non-agricultural forage resource in this analysis. However, vegetation indices that predict spatial and temporal changes in primary productivity and canopy cover (e.g., EVI and normalized difference vegetation index [NDVI]) can be reliable for predicting mast production (Camarero et al. 2010, FernándezMartínez et al. 2015) and were thus used as a proxy.

\section{Crop selection analysis}

Before analyzing the agricultural functional response, we performed a resource selection analysis as an overview of how wild pigs were using crops relative to their availability. In particular, we wanted to ask whether wild pigs were, on average, using crops in proportion to their availability or disproportionately using crops that were less available. This was a third-order resource selection analysis (sensu Johnson 1980) as we were looking at resource selection within a wild pig's established home range.

We used compositional analysis (Aebischer et al. 1993) to compare the proportion of time spent in particular crops to proportional crop availability in terms of crop area. We focused on the dominant crop within the area available to a pig that we defined as the $100 \% \mathrm{MCP}$, with $50-500 \mathrm{~m}$ buffering around the $100 \% \mathrm{MCP}$ to test the sensitivity of the results to our definition of available area. We then tested whether the wild pig used the dominant crop in proportion to its abundance, relative to its use of "other" crops. We considered "other" crops as a single category for this analysis as we were examining average resource selection across wild pigs and different pigs in different regions did not have the same crop types available. In a compositional analysis with two resources, "dominant" and "other" in our case, a single-sample $t$ test determined whether $\ln$ (proportion of dominant used/proportion of other used) - $\ln$ (proportion of dominant available/proportion of other available) was significantly different than zero (Aebischer et al. 1993). We included 122 wild pigs that used two or more crop types over their collaring time in this analysis. For this analysis, the resource use of one wild pig was one data point in the compositional analysis $t$ test.

We also examined 131 wild pigs that used at least one crop type but had two or more crop types "available" 
over their collaring times. For each individual, we ranked available crops by time of use (one being the most used) and by total availability (one being the most available). We then plotted these pig-specific ranks to examine the relationship between rankings of available and used crops across all individuals. Note that the 131 wild pigs included pigs that used at least one resource while the 122 wild pigs in the compositional analysis included pigs that used at least two resources. This was because the compositional analysis used the $\log$ (ratio of use between two resources), which was not well defined when a pig only used one resource. Analyzing rankings of use and availability avoided this issue and allowed for the inclusion of additional pigs in this resource selection analysis.

\section{The shape of the agricultural functional response}

The shape of Type II and Type III functional responses differ for low resource densities, but both are saturating curves as resource density increases (Fig. 1). In contrast, a Type I functional response is linear over all resource densities. Making the assumption that both curves can be approximated by the power-law function consumption rate $=\alpha(\text { resource density })^{\beta}$ at low resource densities, then a Type II functional response should be concave-down with $\beta<1$ and a Type III functional response should be concave-up with $\beta>1$ at low resource densities. A Type I functional response should have $\beta=1$ for all resource densities.

We can use these predictions to test the shape of the agricultural functional response for wild pigs. First, consider resource levels ranging from zero to the resource level at which consumption rate saturates. Fitting a power-law curve to a Type III functional response over this range could produce $\beta<1, \beta=1$, or $\beta>1$ as Type III functional response has concave-up and concavedown portions (Fig. 1). In contrast, a Type II functional response would only yield $\beta<1$. If $\beta<1$ for the functional response, a Type II and Type III functional response could be distinguished by iteratively refitting the power law to a truncated data set, where some percentage of high resource levels are excluded. In this case, we would expect to see $\beta$ increase above 1 for a Type III functional response as we approached the concave-up portion of the functional response at low resource levels and we would expect $\beta$ for a Type II functional response to remain the same or to increase to one, but not above one, at low resource levels. We would expect $\beta$ for a Type I functional response to remain at one for all resource levels.

To test whether the shape of the agricultural functional response was more consistent with a Type I, Type II or Type III response at low crop densities, we assumed that the number of crop visits per month per hour was distributed as a negative binomial, with a parameter $k$ to account for overdispersion in the number of visits ( $k=\infty$ corresponds to a Poisson distribution) and the mean visitation rate $(\lambda /$ collaring time per month) determined by the estimated functional response. To account for the individual-level duration of monitoring, we adjusted the mean number of visits by the collar time such that we were modeling crop visitation rate. We grouped all crop types into a single crop variable for this analysis, such that the data that defined the functional response were the monthly visitation rates to all crop types for a particular wild pig in a given month in a given year. We fit the model

$$
\begin{gathered}
\text { number of crop visit } \sim \text { Negative } \operatorname{Binomial}(\lambda, k) \\
\qquad \begin{array}{c}
\log \left(\frac{\lambda}{\text { collar time per month }}\right)=\alpha \\
+\beta \log (\text { crop area })+(1 \mid \text { pig ID })
\end{array}
\end{gathered}
$$

where $\log ($ crop area $)=\log ($ crop area available/total area available). This model is a power-law function between visitation rate and crop area and $\beta$ is the slope parameter of interest.

We included a random effect of individual, ( $1 \mid$ pigID), to allow individual effects of each wild pig to be explicitly modeled because our data included multiple longitudinal measurements for each wild pig over a given time period. While different individuals may have different shaped functional responses, there were several wild pigs in our dataset that were only collared for two to three months (i.e., two or three data points per individual), making it statistically difficult to estimate individuallevel variation in the shape of the functional response, beyond individual variation in the intercept. We excluded individuals that did not have any available crop area. There were 958 observations and 231 wild pigs to which we fit Eq. 1.

We fit this model six times. The first fit included all 958 observations. The other five fits excluded data points where crop area was greater than the 90th percentile $(n=862)$, the 80th $(n=766)$, the 70th $(n=672)$, the 60 th $(n=578)$, and the 50th $(n=479)$ to focus on the low resource density where Type II and Type III functional responses differ. For each fit we examined the predicted slope $\beta$ and its $95 \%$ credible interval. If our data were consistent with a Type III functional response, we predicted that $\beta$ would increase above one as we increasingly reduced the maximum resource density. In Appendix S1: Section S7 we fit a generalized functional response model (Rosenbaum and Rall 2018) and our results were consistent between the two approaches.

\section{The context dependence of the agricultural functional response}

In our second analysis, we examined how the presence of non-agricultural forage resources affected the agricultural functional response of wild pigs (Fig. 1). As discussed above, we considered two measures of use: monthly visitation rate to crops and the total monthly 
time a particular crop was used. For monthly time spent in crops, we only considered data points for which there was a non-zero time spent in crops because use vs. no use was a statistically distinct process compared to total time of use. To include zeros in our monthly time spent in crops analysis, we would have to use a hurdle model to show use vs. no use and total time spent in crops, conditional on use. However, given that our main interest was total time spent in crops, we focused on the conditional use process of the hurdle model rather than use vs. no use (note that because the likelihoods of the two processes are separable in the hurdle model we can exclude the use vs. no-use model without affecting inference on total time spent in crops). In our analysis of visitation rate, zeros could have emerged naturally from low visitation rates. To be consistent with our analysis of monthly time in crops, however, we also excluded months with zero visits. There were 168 wild pigs and 826 data points to which we fit these models. Note that we used fewer pigs in this model than the agricultural functional shape model in the previous analysis because we excluded pigs that had crops available but never used crops.

Our response variables were either duration of crop use (time spent in crop type per month/total time collared per month) or number of crop visits per month (full model formulation in Appendix S1: Section S8). Our predictor variables were crop group, $\log _{10}$ (crop area available/total area available) $=$ crop area, mean monthly EVI in a wild pig's core home range $=$ EVI, $\log _{10}$ (mean distance from core home range to all available crop fields of particular type) $=$ crop distance, whether or not a crop type was being planted or harvested in a month = crop seasonality, mean canopy cover in a $200-\mathrm{m}$ annulus around all crop fields/mean canopy cover in core home range = canopy cover, and sex. The link function for each model was given by

$$
\begin{aligned}
\eta & =\text { crop group } \times \text { crop area } & & (\text { Agricultural FR }) \\
& + \text { crop group } \times \text { EVI } \times \text { crop area } & & \text { (Non-ag. resopurces }) \\
& + \text { crop group } \times(\text { crop distance } & & \\
& + \text { crop seasonality }) & & \text { crop characteristics }) \\
& + \text { canopy cover } & & \text { (Environmental factors }) \\
& + \text { sex }+(1 \mid \text { pigID }) & & \text { (Pig attributes })
\end{aligned}
$$

Each model tested the hypothesis that non-agricultural forage resources, represented by EVI, affected the agricultural functional response, given by the interaction term in Eq. 2. We $z$-transformed all continuous variables before fitting the model in the probabilistic programming language Stan using a Bayesian framework (Appendix S1: Section S8). We used top-down model selection to initially include all potentially relevant interactions in the model. We then iteratively removed interactions and covariates that reduced model WAIC (Appendix S1: Table S2, S3). We also performed a crossvalidated LASSO regression on the full model in Eq. 2 that shrunk nonsignificant coefficients to zero. The results were consistent with our top-down model selection. We assessed model goodness of fit using $R^{2}$ for generalized linear mixed-effect models in a Bayesian framework (Gelman et al. 2017).

\section{RESULTS}

\section{Crop selection}

Overall, $52 \%$ (168 out of 326 individuals) of wild pigs used 27 different crop types of the 41 crop types available; $29 \%$ (95 individuals) had no crops available, and $19 \%$ (63 individuals) had crops available in their home range but never used them during their collar duration. The most used crops by state in proportion to other available crops, were grapes in California, oranges in Florida, rye in Georgia, sugarcane and corn in Louisiana, soybeans in Missouri and South Carolina, and sorghum in Texas (Appendix S1: Fig. S2). Across all wild pigs, fruit and nut crops had the greatest crop use per hour of collaring time, followed by cereal, cotton, sugar, oilseed, and grasses (Appendix S1: Fig. S3). Vegetables and melon crops, beverage and spice, and roots and tubers were available but not used; and tobacco was not available to wild pigs in this study (Appendix S1: Fig. S3). Of the crops available, wild pigs tended to use crops proportionately to their availability relative to other crops (Fig. 2). On average, they used the dominant crop available in proportion to its availability (Fig. 2A) and the rank of crops available described $90 \%$ of variation in rank of crops used, with a slope of $0.94(95 \% \mathrm{CI}$ about the slope [0.92, 0.97]; Fig. 2B).

\section{The agricultural functional response}

The agricultural functional response for wild pig crop use was consistent with a Type III functional response at low crop availability, with accelerating crop use at low crop availability and evidence for a saturating relationship between crop availability and crop visitation rate at higher crop availability (Fig. 3A,B, Appendix S1: Section S7). When we included the full observed range of crop densities the log-log slope of the functional response had $\beta=1$, inconsistent with a Type II functional response where we would expect $\beta<1$ (Fig. 3B.). As we progressively excluded higher crop densities $\beta$ increased above one, indicating a concave-up relationship between crop use and crop availability that is consistent with a Type III functional response (Fig 3B, Appendix S1: Section S7). The log-log slope $\beta$ was statistically different than one at low crop density, providing evidence against a Type I functional response (Fig. 3).

The presence of non-agricultural forage resources reduced the agricultural functional response in terms of monthly time in crops and the monthly visitation rate to crops (Fig. 4). There was a significant negative interaction between EVI and monthly time in crops and crop visitation rate: when non-agricultural forage was highly 


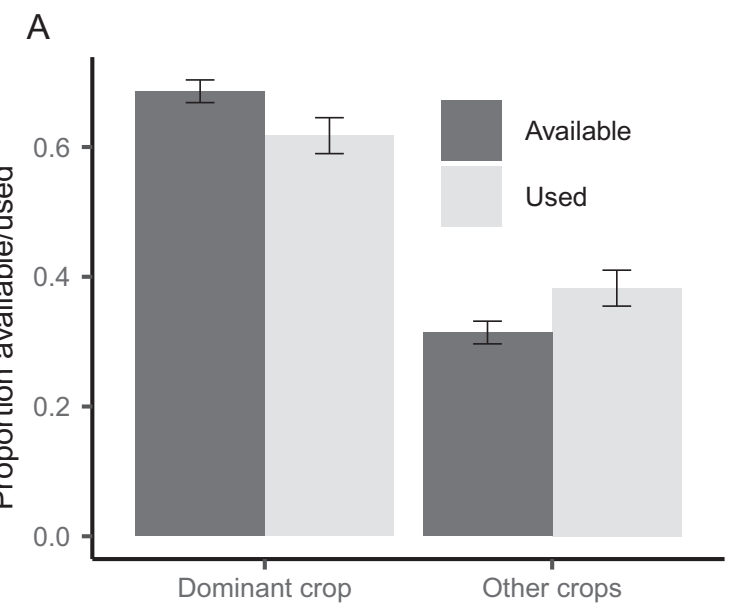

B

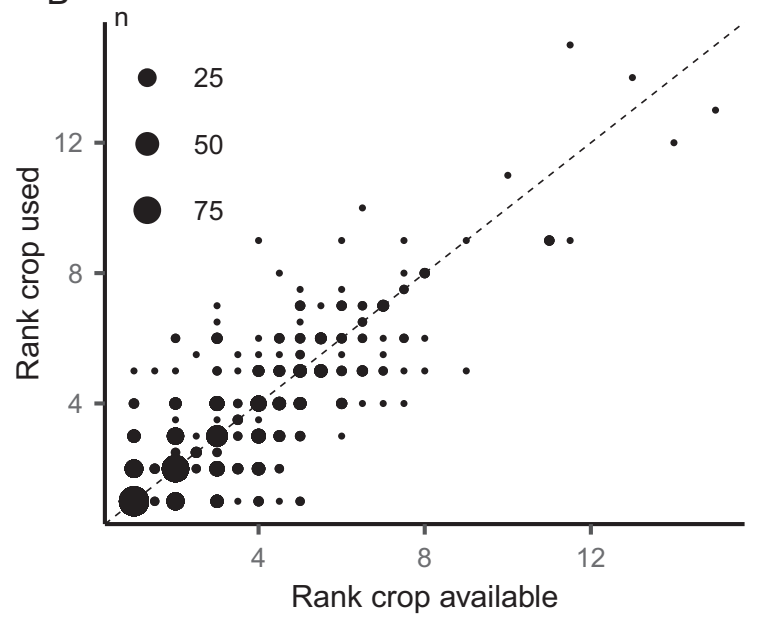

FIG. 2. (A) The average proportional use (measured as time spent in a crop) of the dominant crop available to a wild pig compared to proportional use of other available crops. The average was for 122 wild pigs that had two or more crops available. Error bars are $\pm \mathrm{SE}$ in proportional use/availability. The compositional analysis, where each wild pig was one sample, showed no significant difference between $\log$ (proportional use) and the $\log$ (proportional availability of the dominant crop relative to other crops) $\left(t_{121}=-0.24, P=0.81\right)$. (B) The relationship between rank crops used and rank crop available for 131 wild pigs that used at least one crop and had at least two crops available. The size of the dot indicates the number of points in an area. The rank of the crops available to a wild pig predicted $90 \%$ of the variation in the rank of crops used with a slope of 0.94 (95\% confidence interval $[0.92,0.97])$. The results in panels A and B were unchanged when we buffered the area available to the wild pig by $50-500 \mathrm{~m}$ around the $100 \%$ minimum convex polygon that determined available space (Appendix S1: Section S6).

available, the functional response to increased crop availability was weaker (Appendix S1: Tables S4, S5; Fig. 4). The interactive effect between non-agricultural forage resources and the functional response (namely, the term crop area:EVI in Appendix S1: Tables S4, S5) was independent of crop group ( $\triangle$ WAIC between the model with
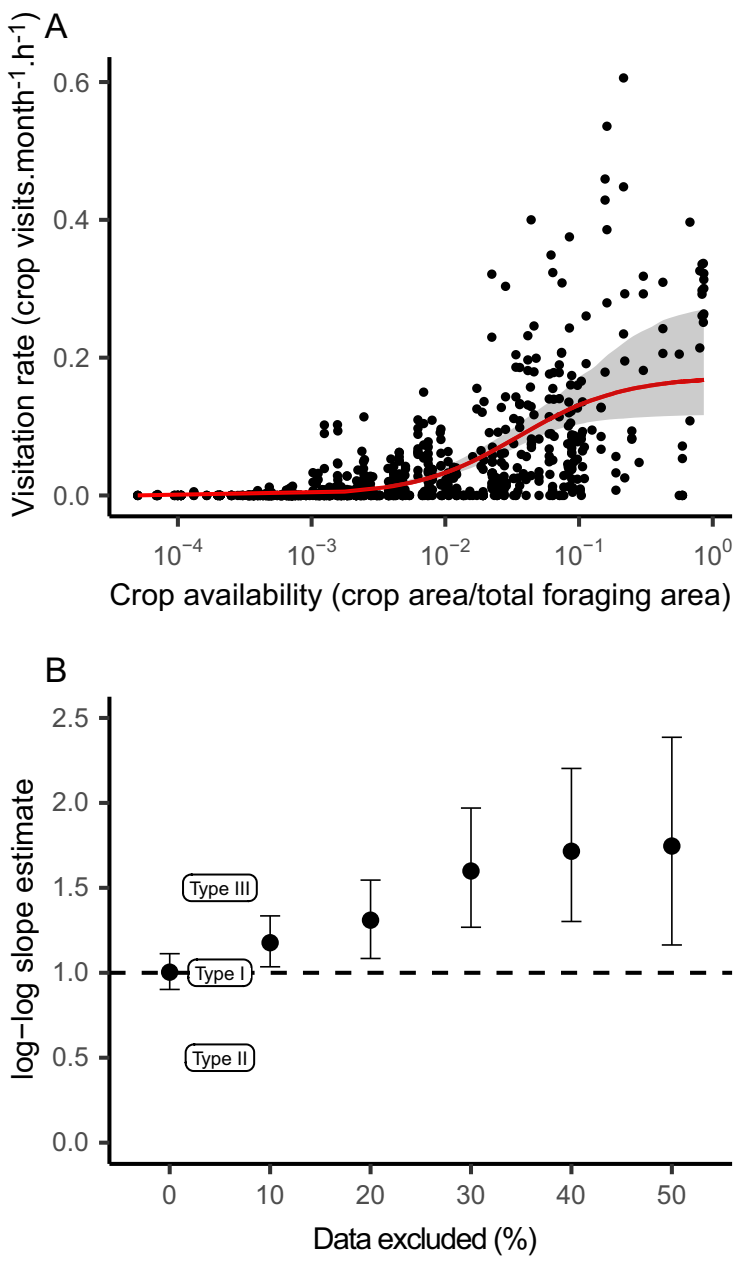

FIG. 3. (A) The agricultural functional response of North American wild pigs. Black points are the observed crop visitation rates for a particular wild pig in a given month in a given year. The red line gives the best fit agricultural functional response using a generalized functional response model (Appendix S1: Section S7). The shaded region is the 95\% credible interval about the median visitation rate. (B) The results of fitting the power-law functional response model given in Eq. 1 to the data shown in A. By iteratively excluding some upper percentile of crop availability (e.g., $10 \%, 20 \%$, etc.) and refitting the model, we would expect the log-log slope (i.e., the powerlaw exponent) of a Type III functional response to increase above 1 . We would expect the log-log slope of a Type II functional response to start below 1 and increase to 1 , but not above 1. Finally, we would expect the slope to remain at one for a Type I functional response. The plot shows the data are consistent with a Type III functional response. This was also confirmed with a generalized functional response model in Appendix S1: Section S7.

a crop group-specific EVI interaction and the best model without a crop group-specific interaction: monthly time in crops model $=\Delta$ WAIC 6.7 ; crop visitation rate model $=\Delta$ WAIC 8.4). In contrast, the agricultural functional response in terms of monthly time of crop use varied by crop group (Appendix S1: Table S4, Fig. S4). 
A
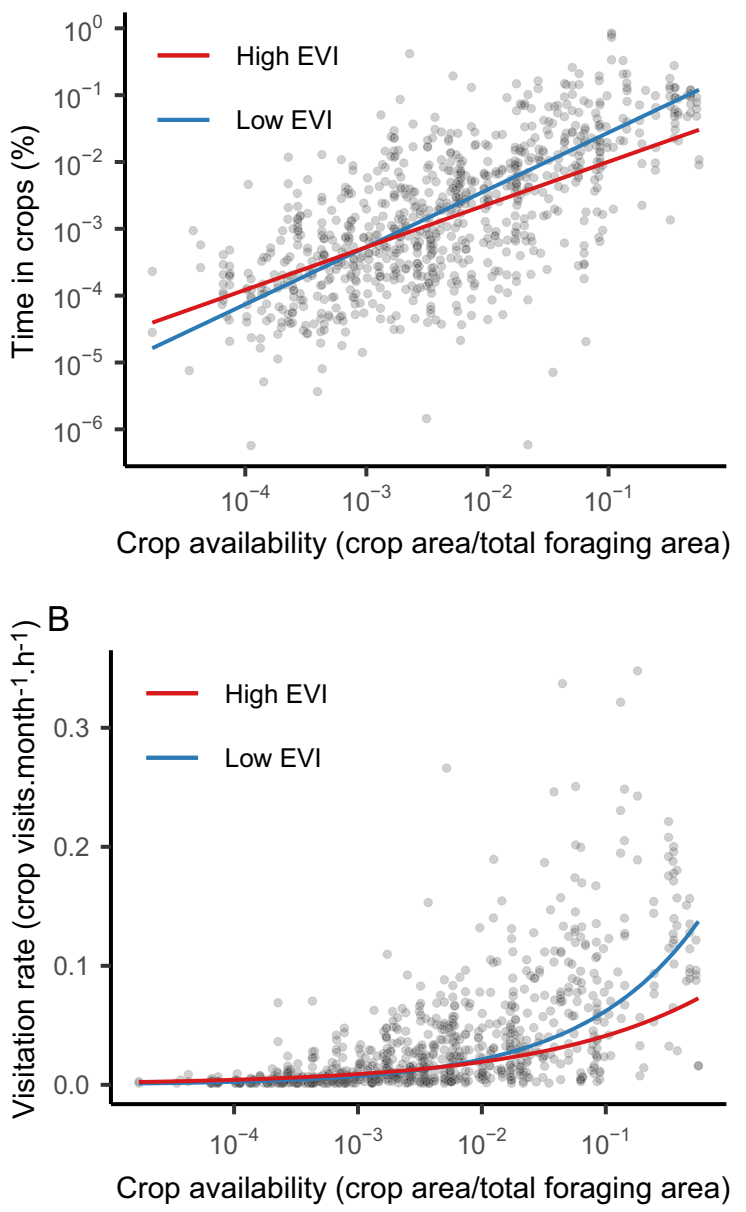

FIG. 4. (A) The effect of non-agricultural resource availability, for which the enhanced vegetation index (EVI) was a proxy, on the agricultural functional response of wild pigs. The gray points are the observed monthly time spent in crops for a particular wild pig in a particular crop group in a given month in a given year. The solid red line (high EVI) and solid blue line (low EVI) line give the predicted agricultural functional response with EVI at the 97.5th percentile and 2.5th percentile of the observed data. (B) The same as panel A, but with crop use defined as the crop visitation rate, i.e., the number of crop visits per month per hour.

Generally, wild pigs showed the smallest mean increase in crop use with a unit increase in crop availability for grasses and cotton (Appendix S1: Fig. S4). As crops became more available, wild pigs generally preferred cereals and fruit and nut crops relative to other crop groups (as indicated by time spent and visitation frequency), given the same amount of area available (Appendix S1: Fig. S4, S5).

Additional crop and wild pig attributes also affected crop use. For every 36\% increase in kilometers from the center of the home range to a crop field, wild pigs reduced the mean percentage of time in crop fields by a median of $13 \%(95 \%$ CI $[6 \%, 20 \%])$ and reduced mean crop visitation rate by a median of $27 \%$ ( $95 \%$ CI $[19 \%$, 35\%]; Appendix S1: Tables S4, S5). When crops were seasonally available, pigs spent a median of $12 \%$ more time in crops $(95 \%$ CI $[1 \%, 23 \%]$; Appendix S1: Table S4) and this effect did not vary by crop group $(\triangle$ WAIC between the two models $=3.9$ : (1) model with crop seasonality and crop group interaction and (2) best fit model without this interaction). Canopy cover around crop fields did not affect either monthly time spent in crops or monthly visitation rate (Appendix S1: Tables S4, S5), but was retained in the best fit models for crop use.

Wild pig identity was an important predictor of crop use, accounting for the second largest amount of variability in crop use after crop area. Excluding identity increased WAIC by 104 and 227 units for monthly time in crops and visitation rate, respectively. Ecologically, this means that individual pigs showed significantly different propensities to use crops. After accounting for wild pig identity, sex also was a significant predictor of crop use, with male wild pigs spending a median of $20 \%$ more time in crops $(95 \%$ CI $[2 \%, 44 \%])$ and showing a $58 \%$ higher crop visitation rate than females $(95 \%$ CI [22\%, 105\%]; Appendix S1: Tables S2, S3). Considering overall goodness of fit, the best agricultural functional response model for monthly time in crops (Appendix S1: Table S2) explained $50 \%$ of variation in crop use through fixed effects only, which increased to $60 \%$ of explained variation when both fixed and random effects were included (Gelman et al. 2017). Similarly, the best agricultural functional response model for visitation rate (Appendix S1: Table S3) explained $44 \%$ of the variation in crop use through fixed effects and $67 \%$ percent of the variation in crop use through fixed and individual identity random effects.

\section{Discussion}

Invasion ecology has long sought to find consistent predictors of invasiveness and damage capacity for IAS (Dick et al. 2014). Functional responses are a promising way forward, but context dependencies and the extrapolation of laboratory-based functional response to field predictions limit functional responses when making predictions in an invasion context (Dick et al. 2014, Vonesh et al. 2017). For wild pigs, an IAS causing significant ecological damage in North America (Mayer and Brisbin 2009), understanding how they use crops is important because it may be a significant contributing factor in their expansion (Lewis et al. 2017, Snow et al. 2017a). Using national-scale wild pig movement data, we found that variability in agricultural functional responses across habitats and individuals can be explained by ecological factors such as the availability of non-agricultural resources, pig attributes, and the stimulus of agricultural resources.

Wild pigs displayed a strong agricultural functional response, with increased availability of crop resource 
predicting an increased use in crop resources. The agricultural functional response in wild pigs was consistent with a Type III functional response, where crop use was an accelerating function of crop availability at low crop densities. Moreover, the agricultural functional response was predictably context-dependent, with pigs responding to increasing crop availability to a lesser extent when non-agricultural forage resources were more available. Taken together, these results suggested wild pigs preferentially used non-agricultural resources over agricultural resources when non-agricultural forage resources were available.

Resource switching is a candidate explanation for the emergence of a Type III agricultural functional response. In a one-consumer-two-resource system, resource switching occurs when a consumer tends to use a resource at disproportionately low rates relative to that resource's availability when at low density, and disproportionately high rates when that resource is at high density (Oaten and Murdoch 1975, Van Leeuwen et al. 2007). Dietary studies suggest wild pigs preferentially forage on non-agricultural resources when available (Sjarmidi et al. 1992, Fournier-Chambrillon et al. 1995) but will switch to primarily feeding on agricultural resources as they become more available (Ballari and Barrios-García 2014). A Type III functional response is even more likely to emerge when one resource is substantially more abundant than another in a two-resource system (Van Leeuwen et al. 2007). Non-agricultural resources may be more consistently available due to factors such as crop seasonality, the distance needed to travel to forage on crops and crop protection practices, further promoting the emergence of a Type III agricultural functional response. Resource switching can be driven by a consumer learning to more efficiently exploit a resource as encounters between consumer and resource increase (Murdoch and Oaten 1975). Studies have shown wild pigs can remember locations of profitable resources and adjust their behavior to exploit these resources (Held et al. 2005), potentially promoting a Type III functional response.

Four general factors affect the shape of the functional response for a focal resource: non-focal resource availability, focal resource characteristics, consumer attributes, and environmental variables (Holling 1959, Dick et al. 2014). As omnivores, wild pigs feed on whatever resources are present (Mayer and Brisbin 2009, Lavelle et al. 2017), but their population dynamics can be strongly influenced by pulsed resources (i.e., mast seeding; Bieber and Ruf 2005) motivating our prediction that the agricultural functional response in wild pigs would change as non-agricultural resources became more available. Similarly, we found that, as non-agricultural forage resources became more abundant within a wild pig's core home range, the agricultural functional response in terms of monthly crop use decreased disproportionately more in areas with high crop availability relative to areas with low crop availability. In other words, when non-agricultural forage resources were highly available, wild pigs responded less to changes in crop availability. The interactive effect of non-agricultural resource availability on monthly crop use was driven by two factors: monthly crop visitation rate and average time in crops per visit (Appendix S1: Section S5), both of which also showed negative interactions between crop availability and non-agricultural resource availability.

Crop resource attributes affected the agricultural functional response beyond crop availability. The distance to crops can be generalized as the stimulus a resource provides to a consumer (Holling 1959) and we found that decreasing the resource stimulus (i.e., increasing the distance from crops) reduced the magnitude of the functional response. We also found wild pigs increased time spent in crops when crops were seasonally available, consistent with previous studies in North America and Europe (Ballari and BarriosGarcía 2014, Engeman et al. 2018, Paolini et al. 2018). Finally, the agricultural functional response varied depending on crop type. For example, it was generally weaker with respect to grasses and cotton crops (i.e., a reduced slope) compared to cereals. In terms of calories alone, grass crops and cotton have a lower caloric content than most cereal crops that were heavily used (alfalfa $[$ grass] $=194 \mathrm{kcal} / 100 \mathrm{~g}$; cotton $=180 \mathrm{kcal} / 100$ $\mathrm{g}$; corn $[$ cereal] $=365 \mathrm{kcal} / 100 \mathrm{~g}$; sorghum $[$ cereal] $=$ $329 \mathrm{kcal} / 100 \mathrm{~g}$; rye [cereal] $=338$; USDA 2018b). While the increased functional response slope for cereals compared to grasses and cotton may reflect increased caloric values of cereals, calories alone ignore other nutritional and non-nutritional aspects of a crop. For example, certain agricultural crops may also provide seasonal water, cover, refuge, or thermoregulation opportunities, and crop production practices can alter soil productivity via soil amelioration or nutrient input as methods to promote crop growth that may also tangentially increase other favorable herbaceous forage and crop root pests such as beetle larvae, which may promote their selection and increase time spent in the crops. Generally, when comparing linear or log-linear agricultural functional responses, slope is likely a more robust measure of resource preference than the magnitude of the agricultural functional response, as the magnitude is also influenced by the intercept that accounts for intrinsic properties of the resource that affects the amount of resource use (e.g., how hard it is to move through the resource), beyond resource preference.

Individual-level heterogeneity is an important driver of the variance in behavior and landscape use in wildlife (Morales and Ellner 2002, Patterson et al. 2008). We identified two consumer characteristics, wild pig identity and sex, that affected the agricultural functional response. Given that wild pigs have a variable capacity to learn (Held et al. 2005), were of different ages in this study, and were living in different environments, the importance of individual identity in describing variability in the agricultural functional response was not 
surprising. Sex, on the other hand, was an important predictor of crop use, with subadult/adult male wild pigs spending more time in crops and visiting crops more than females, after accounting for individual-level variability. Previous studies on wild pig movement in North America found males generally moved more than females, and that females in matriarchal social groups (i.e., sounders) generally showed high site fidelity whereas dispersed and mature males were less risk averse regarding novel food sources than females, all of which could promote higher contact with crop fields for males (Sparklin et al. 2009, Kay et al. 2017, Lavelle et al. 2018). This was supported by our finding of no difference between males and females in terms of average length of crop visits (Appendix S1: Section S5), suggesting visitation rates were driving differences in monthly crop use between sexes. Increased contact with crop fields could promote increased efficiency of crop depredation, suggesting identifying characteristics of animals that correlate with increased visitation rates can help target individuals that are more risk tolerant (i.e., males that are less risk averse).

Canopy cover around crop fields, the single environmental factor that we examined in this analysis, did not alter the agricultural functional response despite previous studies indicating that increased canopy cover would provide refuge from which wild pigs could raid crop fields (Amici et al. 2012). While the National Land Cover Database (NLCD) canopy cover layer depicts the tree canopy, it does not include understory species that are similarly important for providing cover and aiding with thermoregulation for wild pigs (Mayer and Brisbin 2009). Including an additional environmental variable of understory density could be an important factor for predicting the agricultural functional response. However, in accordance with previous studies, wild pigs in our study consistently spent more time in areas with high canopy cover, reinforcing that cover is an important component of suitable wild pig habitat both as thermal and hiding cover from predators (Appendix S1: Fig. S6; Mayer and Brisbin 2009). Canopy cover may indirectly affect crop use by determining where an invading wild pig establishes, which may place it in close proximity to agricultural resources.

Individual-level foraging behaviors inform functional responses that, in turn, shape population-level dynamics (Murdoch and Oaten 1975). The reduction in agricultural functional response with increasing nonagricultural forage resources suggests potential individual-level foraging mechanisms driving resource use in wild pigs. Comparing our results with predictions from foraging theory can help us understand these mechanisms. All else equal, the marginal value theorem of optimal foraging theory predicts average visit length in a resource patch should decrease as the mean resource level of patches on the landscape increases (Charnov 1976). In this context, wild pigs should spend less time per visit in crop fields when non-agricultural resources are increasingly available, what we observed, as they need to acquire less nutrients from crops to meet their maintenance requirements because they are spending more time in non-agricultural resources.

In contrast, when limited by foraging time and resource patches on a landscape are variable, risk-averse foragers should prefer the less variable resource, even if the expected net nutrient gain from the more variable resource is higher (Caraco 1980, 2012). Despite being nutritionally rich, some crop resources are potentially more variable than non-agricultural resources in their availability (e.g., field conversion) and the nutrient composition required for wild pigs to select for them, as exploiting crops can require long-distance travel and energetic risks such as increased predation when leaving areas of high cover and increased injury or mortality through crop protection methods (Keuling et al. 2009, Mayer and Brisbin 2009). The optimal strategy may be to reduce visitation rates to less variable and less risky non-agricultural forage resources, which is also consistent with what we observed. While crops are not universally more variable and/or riskier to exploit for wildlife compared to non-agricultural resources, many strategies for deterring or preventing wildlife from exploiting crops are energetically costly for the targeted species (e.g., scaring, toxic baiting, shooting, and constructing barriers; Craven and Hygnstrom 1994, Snow et al. 2017b). Taken together, our results suggest multiple individual-level foraging mechanisms may describe how availability of non-agricultural resources altered the magnitude of the agricultural functional response in wild pigs.

The damage an IAS inflicts on a resource is directly related to their functional response to the availability of that resource (Dick et al. 2014). However, the quantitative link between the observed functional response and predicted damage is still unclear (Vonesh et al. 2017). Our research is the first to use national-level movement data to link space use of a large invasive mammal to total time spent in crops and functional responses, particularly in agro-ecosystems. The total time spent in crops could be a useful proxy for quantifying crop damage by wildlife as it likely captures direct damage through resource consumption and indirect damage such as trampling or rooting. There are a variety of existing approaches for assessing crop damage by wildlife such as in-the-field measurements (e.g., Engeman et al. 2018) and farmer and drone surveys (Anderson et al. 2016, Michez et al. 2016). Our approach provides a potential alternative approach to assessing crop damage where the resulting functional response model could be used to retroactively estimate crop damage from where an animal has spent time and predict crop damage in an area where an animal has yet to establish. An important next step before using the results of this study to predict damage, however, will be to link field-level measures of damage to the GPS movements of an animal. An additional challenge in predicting potential crop damage where an IAS has yet to establish is first determining the 
likely core home range and available space of that IAS. Home-range size analyses (Garza et al. 2017) and resource selection functions (Manly et al. 2007) could be used together to predict the likely size and location of animal home ranges, from which the functional response model could account for non-focal resource availability, focal resource characteristics, consumer attributes, and environmental variables to predict crop use and damage.

\section{ACKNOWLEDGMENTS}

We thank the researchers, technicians, and volunteers who contributed to the fieldwork. Thanks to Deedra Murrieta, Tyler Campbell, Susan Cooper, Michael Tabak, and David Wolfson for their help with GIS and GPS data and their constructive comments. The research was supported in part by the USDA, US DOE-Savannah River Operations Office through the USDA Forest Service-Savannah River under Interagency Agreement DE-AI09-00SR22188, USDA APHIS Agreement 12-7412-0896-CA to S. M. Wisely, USDA Center for Epidemiology and Animal Health cooperative agreements \#9200-0391 from 2014-2018 to R. K. Boughton. Contributions of J. C. Beasley and S. M. Chinn were partly supported by the US DOE (DE-FC09-07SR22506 to the University of Georgia Research Foundation). All trapping and handling of wild pigs followed the guidelines established in an approved animal use protocol, IACUC Protocol \#16059 (University of California, Davis) and approval for trapping, collaring, marking, and releasing of wild pigs in California followed the guidelines established in an approved scientific collecting permit (California Department of Fish and Wildlife [CDFW] Scientific Collecting Permit \#11225), \#201308160, 201408495 \& 201808495 (University of Florida), QA-2632 \& QA-2724 Camp Bullis), PRN 2003-0531 \& 2007-1196 (Auburn University), SREL protocol A2012 08004-Y3-A5. Any use of trade, firm, or product names is for descriptive purposes only and does not imply endorsement by the U.S. Government. MQW, SMC, JCB, RB, RSM, CTW, KMP conceived and designed methodology. S M. Chinn, J. C. Beasley, R. Brook, R. K. Boughton, S. S. Ditchkoff, J. W. Fischer, S. B. Hartley, L. K. Holmstrom, J. C. Kilgo, J. S. Lewis, R. S. Miller, N. P. Smow, K. C. VerCateren, and S. M. Wisely collected the field data. M. Q. Wilber and S. M. Chinn conducted data analyses and interpretation. M. Q. Wilber and S. M. Chinn led the writing of the manuscript. All authors contributed critically to the drafts and gave final approval for publication.

\section{Literature Cited}

Aebischer, N. J., P. A. Robertson, and R. E. Kenward. 1993. Composition analysis of habitat use from animal radiotracking data. Ecology 74:1313-1325.

Amici, A., F. Serrani, C. M. Rossi, and R. Primi. 2012. Increase in crop damage caused by wild boar (Sus scrofa L.): The "refuge effect". Agronomy for Sustainable Development 32:683-692.

Anderson, A., C. Slootmaker, E. Harper, J. Holderieath, and S. A. Shwiff. 2016. Economic estimates of feral swine damage and control in 11 US states. Crop Protection 89:89-94.

Ballari, S. A., and M. N. Barrios-García. 2014. A review of wild boar Sus scrofa diet and factors affecting food selection in native and introduced ranges. Mammal Review 44:124-134.

Barrios-García, M. N., and S. A. Ballari. 2012. Impact of wild boar (Sus scrofa) in its introduced and native range: A review. Biological Invasions 14:2283-2300.

Bevins, S. N., K. Pedersen, M. W. Lutman, T. Gidlewski, and T. J. Deliberto. 2014. Consequences associated with the recent range expansion of nonnative feral swine. BioScience 64:291299.

Bieber, C., and T. Ruf. 2005. Population dynamics in wild boar Sus scrofa: ecology, elasticity of growth rate and implications for the management of pulsed resource consumers. Journal of Applied Ecology 42:1203-1213.

Bond, M. L., C. Bradley, and D. E. Lee. 2016. Foraging habitat selection by California spotted owls after fire. Journal of Wildlife Management 80:1290-1300.

Brook, R. K., and F. M. Van Beest. 2014. Feral wild boar distribution and perceptions of risk on the central Canadian prairies. Wildlife Society Bulletin 38:486-494.

Buderman, F. E., M. B. Hooten, J. S. Ivan, and T. M. Shenk. 2016. A functional model for characterizing long-distance movement behaviour. Methods in Ecology and Evolution 7:264-273.

Camarero, J. J., J. Albuixech, R. Lopez-Lozano, M. A. Casterad, and G. Montserrat-Marti. 2010. An increase in canopy cover leads to masting in Quercusilex. Trees 24:909-918.

Caraco, T. 1980. On foraging time allocation in a stochastic environment. Ecology 61:119-128.

Caraco, T. 2012. Foraging Behavior. Pages 1-823 in A. Hastings and L. J. Gross, editors. Encyclopedia of theoretical ecology. University of California Press, Berkeley, California, USA.

Charnov, E. L. 1976. Optimal foraging theory: the marginal value theorem. Theoretical Population Biology 9:129-136.

Craven, S. R., and S. E. Hygnstrom. 1994. Deer. Pages D25D40 in S. E. Hygnstrom, R. M. Timm, and G. E. Larson, editors. Prevention and control of wildlife damage. Great Plains Agricultural Council, University of Nebraska, Lincoln, Nebraska, USA.

Dick, J. T. A., et al. 2014. Advancing impact prediction and hypothesis testing in invasion ecology using a comparative functional response approach. Biological Invasions 16:735753.

Dick, J. T. A., et al. 2017. Functional responses can unify invasion ecology. Biological Invasions 19:1667-1672.

Engeman, R. M., J. Terry, L. R. Stephens, and K. S. Gruver. 2018. Prevalence and amount of feral swine damage to three row crops at planting. Crop Protection 112:252-256.

Fernández-Martínez, M., M. Garbulsky, J. Peñuelas, G. Peguero, and J. M. Espelta. 2015. Temporal trends in the enhanced vegetation index and spring weather predict seed production in Mediterranean oaks. Plant Ecology 216:10611072.

Fournier-Chambrillon, C., D. Maillard, and P. Fournier. 1995. Diet of the wild boar (Sus scrofa L.) inhabiting the Montpellier garrigue. Journal of Mountain Ecology (Ibex) 3:174-179.

Garza, S. J., M. A. Tabak, R. S. Miller, M. L. Farnsworth, and C. L. Burdett. 2017. Abiotic and biotic influences on homerange size of wild pigs (Sus scrofa). Journal of Mammalogy 99:1-11.

Gelman, A., B. Goodrich, J. Gabry, and I. Ali. 2017. R-squared for Bayesian regression models. http://www.stat.columbia. edu/gelman/research/unpublished

Godvik, I. M. R., L. E. Loe, J. O. Vik, V. Veiberg, R. Langvatin, and A. Mysterud. 2009. Temporal scales, trade-offs, and functional responses in red deer habitat selection. Ecology 90:699-710.

Held, S., J. Baumgartner, A. Kilbride, R. W. Byrne, and M. Mendl. 2005. Foraging behaviour in domestic pigs (Sus scrofa): Remembering and prioritizing food sites of different value. Animal Cognition 8:114-121.

Holling, C. S. 1959. The components of predation as revealed by a study of small-mammal predation of the European Pine Sawfly. Canadian Entomologist 91:293-320. 
Hooten, M. B., and D. S. Johnson. 2017. Basis function models for animal movement. Journal of the American Statistical Association 112:578-589.

Hooten, M. B., D. S. Johnson, B. T. McClintock, and J. M. Morales. 2017. Animal movement: statistical models for telemetry data. CRC Press, New York, New York, USA.

Johnson, D. H. 1980. The comparison of usage and availability measurements for evaluating resource preference. Ecology 61:65-71.

Kay, S. L., et al. 2017. Quantifying drivers of wild pig movement across multiple spatial and temporal scales. Movement Ecology 5:1-15.

Keuling, O., N. Stier, and M. Roth. 2009. Commuting, shifting or remaining? Different spatial utilisation patterns of wild boar Sus scrofa L. in forest and field crops during summer. Mammalian Biology 74:145-152.

Lavelle, M. J., N. P. Snow, J. W. Fischer, J. M. Halseth, E. H. VanNatta, and K. C. VerCauteren. 2017. Attractants for wild pigs: current use, availability, needs, and future potential. European Journal of Wildlife Research 63.

Lavelle, M. J., N. P. Snow, J. M. Halseth, E. H. VanNatta, H. N. Sanders, and K. C. VerCauteren. 2018. Evaluation of movement behaviors to inform toxic baiting strategies for invasive wild pigs (Sus scrofa). Pest Management Science 74:2504-2510.

Lewis, J. S., M. L. Farnsworth, C. L. Burdett, D. M. Theobald, M. Gray, and R. S. Miller. 2017. Biotic and abiotic factors predicting the global distribution and population density of an invasive large mammal. Scientific Reports 7:1-12.

Lonsdale, W. M. 1999. Global patterns of plant invasions and the concept of invasibility. Ecology 80:1522-1536.

Manly, B. F. J., L. L. McDonald, D. L. Thomas, T. L. McDonald, and W. P. Erickson. 2007. Resource selection by animals: statistical design and analysis for field studies. Second Edition. Chapman and Hall, London.

Mayer, J. J., and J. C. Beasley. 2017. Wild pigs. in W. C. Pitt, J. C. Beasley, and G. W. Witmer, editors. Ecology and management of terrestrial vertebrate invasive species. CRC Press, Boca Raton, Florida, USA

Mayer, J. J., and I. J. Brisbin, Jr.. 2009. Wild pigs: biology, damage, control techniques and management. No. SRNL-RP2009-0086. Savannah River Site (SRS), Aiken, South Carolina, USA.

Michez, A., K. Morelle, F. Lehaire, J. Widar, M. Authelet, C. Vermeulen, and P. Lejeune. 2016. Use of unmanned aerial system to assess wildlife (Sus scrofa) damage to crops (Zea mays). Journal of Unmanned Vehicle Systems 4:266-275.

Morales, J. M., and S. P. Ellner. 2002. Scaling up animal movements in heterogeneous landscapes: the importance of behavior. Ecology 83:2240-2247.

Murdoch, W. W., and A. Oaten. 1975. Predation and population stability. Advances in Ecological Research 9:1-131.

Mysterud, A., and R. A. Ims. 1998. Functional responses in habitat use: availability influences relative use in trade-off situations. Ecology 79:1435-1441.

Northrup, J. M., M. B. Hooten, C. R. Jr Anderson, and G. Wittemyer. 2013. Practical guidance on characterizing availability in resource selection functions under a use-availability design. Ecology 94:1456-1463.

Oaten, A., and W. W. Murdoch. 1975. Switching, functional response, and stability in predator-prey systems. American Naturalist 109:299-318.

Paolini, K. E., B. K. Strickland, J. L. Tegt, K. C. VerCauteren, and G. M. Street. 2018. Seasonal variation in preference dictates space use in an invasive generalist. PLoS ONE 13:e0199078.

Paterson, R. A., J. T. A. Dick, D. W. Pritchard, M. Ennis, M. J. Hatcher, and A. M. Dunn. 2015. Predicting invasive species impacts: A community module functional response approach reveals context dependencies. Journal of Animal Ecology 84:453-463.

Patterson, T. A., L. Thomas, C. Wilcox, O. Ovaskainen, and J. Matthiopoulos. 2008. State-space models of individual animal movement. Trends in Ecology \& Evolution 23: 87-94.

Pepin, K. M., D. W. Wolfson, R. S. Miller, M. A. Tabak, N. P. Snow, K. C. VerCauteren, and A. J. Davis. 2019. Accounting for heterogeneous invasion rates reveals management impacts on the spatial expansion of an invasive species. Ecosphere 10: e02657.

Pettorelli, N., J. O. Vik, A. Mysterud, J. M. Gaillard, C. J. Tucker, and N. C. Stenseth. 2005. Using the satellite-derived NDVI to assess ecological responses to environmental change. Trends in Ecology and Evolution 20:503-510.

Pettorelli, N., S. Ryan, T. Mueller, N. Bunnefeld, B. Jedrzejewska, M. Lima, and K. Kausrud. 2011. The normalized difference vegetation index (NDVI): unforeseen successes in animal ecology. Climate Research 46:15-27.

Pimental, D. 2007. Environmental and economic costs of vertebrate species invasions into the United States. Pages 1-8 in G. W. Witmer, W. C. Pitt, and K. A. Fagerstone, editors. Managing vertebrate invasive species. USDA National Wildlife Research Center Symposia. Fort Collins, Colorado, USA.

Putman, R. J., and N. P. Moore. 1998. Impact of deer in lowland Britain on agriculture, forestry and conservation habitats. Mammal Review 28:141-164.

Rosenbaum, B., and B. C. Rall. 2018. Fitting functional responses: direct parameter estimation by simulating differential equations. Methods in Ecology and Evolution 9:20762090.

Shwiff, S., S. Shwiff, J. Holderieath, W. Haden-Chomphosy, and A. Anderson. 2012. Economics of invasive species damage and damage management. Pages 35-59 in W. C. Pitt, J. C. Beasley, and G. C. Witmer, editors. Ecology and management of terrestrial vertebrate invasive species in the United States. CRC Press, Boca Raton, Florida, USA.

Sjarmidi, A., F. Spitz, and G. Valet. 1992. Food resource use by wild boar in southern France. Pages 171-173 in G. Janeau, G. Gonzalez, and S. Aulagnier, editors. Ongules/Ungulates 91: Proceedings of the international symposium. Tolouse, France.

Snow, N. P., M. A. Jarzyna, and K. C. VerCauteren. $2017 a$. Interpreting and predicting the spread of invasive wild pigs. Journal of Applied Ecology 54:2022-2032.

Snow, N. P., J. A. Foster, J. C. Kinsey, S. T. Humphrys, L. D. Staples, D. G. Hewitt, and K. C. VerCauteren. 2017b. Development of toxic bait to control invasive wild pigs and reduce damage. Wildlife Society Bulletin 41:256-263.

Sparklin, B. D., M. S. Mitchell, L. B. Hanson, D. B. Jolley, and S. S. Ditchkoff. 2009. Territoriality of feral pigs in a highly persecuted population on Fort Benning, Georgia. Journal of Wildlife Management 73:497-502.

Thurfjell, H., J. P. Ball, P.-A. Åhlén, P. Kornacher, H. Dettki, and K. Sjöberg. 2009. Habitat use and spatial patterns of wild boar Sus scrofa (L.): agricultural fields and edges. European Journal of Wildlife Research 55:517-523.

Tolleson, D. R., W. E. Pinchak, D. Rollins, and L. J. Hunt. 1995. Feral hogs in the rolling plains of Texas: perspectives, problems, and potential. Page 454 in R. E. Masters and J. G. Huggins, editors. Proceedings of the 12th Great Plains Wildlife Damage Control Workshop. University of Nebraska, Lincoln, Nebraska, USA.

USDA. 2010. Field crops: Usual planting and harvesting dates, October 2010. USDA, Washington, D.C., USA. 
USDA. 2018a. USDA National Agricultural Statistics Service cropland data layer: published crop-specific data layer. USDA, Washington, D.C., USA.

USDA. 2018b. USDA food composition database. https:// ndb.nal.usda.gov/ndb

Van Leeuwen, E., V. A. A. Jansen, and P. W. Bright. 2007. How population dynamics shape the functional response in a onepredator-two-prey system. Ecology 88:1571-1581.
Vitousek, P. M., C. M. D’Antonio, L. L. Loope, M. Rejmänek, and R. Westbrooks. 1997. Introduced species: a significant component of human-caused global change. New Zealand Journal of Ecology 21:1-16.

Vonesh, J., M. McCoy, R. Aletwegg, P. Landi, and J. Measey. 2017. Functional responses can’t unify invasion ecology. Biological Invasions 19:1673-1676.

\section{SUPPORTING INFORMATION}

Additional supporting information may be found online at: http://onlinelibrary.wiley.com/doi/10.1002/eap.2015/full

\section{DATA ACCESSIBILITY}

Data are available from the Dryad Digital Repository: https://doi.org/10.5061/dryad.k3q6v8j. 